\title{
QUALIDADE DE VIDA, SINTOMAS DEPRESSIVOS E ADESÃO AO TRATAMENTO DE PESSOAS COM HIPERTENSÃO ARTERIAL
}

Jadiel Fellipe Santana Santos ${ }^{1}$, Ana Carolline Rodrigues Lima ${ }^{1}$, Clarissa Maria Dias Mota²,

Cristiane Franca Lisboa Gois ${ }^{3}$, Gabriela Menezes Gonçalves de Brito ${ }^{4}$, Íkaro Daniel de Carvalho Barreto ${ }^{5}$

Objetivo: avaliar a qualidade de vida (OV), sintomas depressivos e adesão ao tratamento de pessoas com Hipertensão Arterial Sistêmica (HAS). Metodologia: estudo do tipo descritivo exploratório, de corte transversal e abordagem quantitativa, realizado com a população de 155 usuários. Foram utilizados quatro instrumentos para a coleta de dados: um para caracterização sóciodemográfica e clínica, o Medical Outcomes Study 36 (SF-36), o Inventário de Depressão de Beck (BDI) e a Medida de Adesão aos Tratamentos (MTA). Os dados foram analisados segundo testes estatísticos e regressão linear simples. Resultados: o domínio mais comprometido da OV foi Aspecto físico (Média = 49,0); 34,8\% apresentaram disforia, e 14,2\% depressão moderada; 76,8\% aderiam ao tratamento. Conclusão: as atividades avaliadas no domínio Aspecto físico da OV foram as mais comprometidas. A maioria dos sujeitos apresentou alteração psicológica e aderiu ao tratamento medicamentoso.

Descritores: Qualidade de vida; Hipertensão; Depressão; Adesão à medicação.

\section{QUALITY OF LIFE, DEPRESSIVE SYMPTOMS AND ADHERENCE TO TREATMENT OF PEOPLE WITH HYPERTENSION}

Objective: to evaluate the quality of life ( $\mathrm{OOL}$ ), depressive symptoms and adherence to treatment of people with Hypertension. Methodology: the study type is descriptive exploratory, cross-sectional and quantitative approach, with a total of 155 user as a target population. For data collection, four instruments were used: one for sociodemographic and clinical characterization, the Medical Outcomes Study 36 (SF-36), the Beck Depression Inventory (BDI) and the Measurement of Treatment Adherence (MTA). Data analyzes was performed with statistic tests and linear regression. Results: the most compromised domain of OOL was physical aspect (mean $=49.0$ ); $34.8 \%$ of subjects had dysphoria, and $14.2 \%$ moderate depression; $76.8 \%$ had adhered to treatment. Conclusion: the activities evaluated in the physical domain of QOL were the most compromised. Most of subjects showed psychological change and adhere to drug treatment.

Descriptors: Quality of life; Hypertension; Depression; Medication adherence.

\section{CALIDAD DE VIDA, SÍNTOMAS DEPRESIVOS Y LAADHESIÓN A LAS PERSONAS CON TRATAMIENTO DE LA HIPERTENSIÓN}

Objetivo: evaluar la calidad de vida (CV), síntomas depresivos y la adherencia al tratamiento de las personas con hipertensión arterial sistêmica (HAS). Metodología: estudio exploratorio descriptivo, transversal y de enfoque cuantitativo, realizado con 155 usuarios. Se utilizaron cuatro instrumentos para la recolección de datos: un para la caracterización sociodemográfica y clínica, el Medical Outcomes Study 36 (SF-36), el Inventario de Depresión de Beck (BDI) y la Medición de la adherencia al tratamiento (MTA). Para analisis de los datos se realizaron testes estadisticos y análisis de regresión lineal. Resultados: La zona más afectada de la CV fue el aspecto físico (media = 49,0); 34.8\% tenían disforia, y el 14,2\% depresión moderada; 76,8\% adhiere al tratamiento. Conclusión: las actividades evaluadas en el dominio de aspecto físico de CV son los más comprometida. La mayoría mostró cambio psicológico y se adhieren al tratamiento farmacológico.

Descriptores: Calidad de vida; Hipertensión; Depresión; Adherencia a la medicación.

${ }^{1}$ Enfermeiro. Universidade Federal de Sergipe, UFS

${ }^{2}$ Enfermeira. Mestranda. Universidade Federal de São Paulo, UNIFESP.

${ }^{3}$ Enfermeira. Doutora em Enfermagem. Professora da UFS.

${ }^{4}$ Enfermeira. Doutoranda em Enfermagem. Universidade Federal da Bahia. Email: Gabriela.menezes.g@hotmail.com

${ }^{5}$ Estatistico. Mestre em Estatistica. Universidade Federal de Pernambuco, UFPE. 


\section{ARTIGO 3}

QUALIDADE DE VIDA, SINTOMAS DEPRESSIVOS E ADESÃO AO TRATAMENTO DE PESSOAS COM HIPERTENSÃO ARTERIAL Jadiel Fellipe Santana Santos, Ana Carolline Rodrigues Lima, Clarissa Maria Dias Mota, Cristiane Franca Lisboa Gois, Gabriela Menezes Gonçalves de Brito, İkaro Daniel de Carvalho Barreto

\section{INTRODUÇÃO}

A Hipertensão Arterial Sistêmica (HAS) é uma doença crônica que apresenta elevada morbimortalidade e constitui um dos principais fatores de risco modificáveis para as doenças cardiovasculares ${ }^{(1)}$

Em 2014, o Brasil apresentava cerca de 126 milhões de pessoas com HAS cadastradas; dessas, em torno de 100 milhões residiam na zona urbana. No mesmo periodo, foram registradas pouco mais de um milhão de internações por doenças do aparelho circulatório; dessas, em torno de 75 mil por complicações decorrentes da HAS essencial ou primária, o que representou a sexta maior causa de internação entre as doenças do aparelho circulatório, gerando um gasto de aproximadamente 26 milhões de reais aos cofres públicos ${ }^{(2)}$.

As mudanças impostas pela doença crônica na vida das pessoas podem se refletir negativamente na qualidade de vida $(\mathrm{OV})^{(3)}$. No contexto do indivíduo com HAS, os domínios da qualidade de vida que têm sido identificados como mais comprometidos são Capacidade funcional(4). Aspectos físicos ${ }^{(5)}$ e Vitalidade ${ }^{(6)}$.

Além disso, estudos também têm pesquisado a relação da OV com a depressão(5), assim como com a adesão ao tratamento medicamentoso(7). A depressão pode interferir negativamente na OV de pessoas com HAS ${ }^{(5)}$, como também a baixa adesão ao tratamento pode estar associada à pior OV e à depressão(7).

Resultado de um estudo de revisão sistemática, que teve como objetivo examinar a força e a consistência das evidências sobre a relação entre depressão e adesão aos medicamentos anti-hipertensivos, concluiu que, embora os estudos tenham revelado existir relação entre depressão e baixa adesão, não é possivel concluir definitivamente essa relação em virtude da heterogeneidade dos estudos, no que diz respeito à avaliação da depressão e adesão, bem como das inconsistências nos resultados. ${ }^{(8)}$.

Assim, considerando a elevada prevalência e morbidade associada à HAS, a relação entre QV, depressão e adesão ao tratamento, o número reduzido de artigos desenvolvidos com essa temática, o presente estudo teve como objetivo avaliar a OV, a presença de sintomas depressivos e a adesão ao tratamento medicamentoso de pessoas com HAS acompanhadas por uma Equipe de Saúde da Família (ESF) do município de Aracaju, estado de Sergipe.

\section{METODOLOGIA}

Estudo descritivo, exploratório, de corte transversal e abordagem quantitativa realizado com pessoas com HAS, acompanhadas na Atenção Primária de Saúde, no ano de 2012.

A população foi constituída por todas as pessoas/ usuários acompanhados por uma ESF do município de Aracaju, que atenderam aos seguintes critérios de inclusão: idade igual ou superior a 18 anos, possuir indicação médica para o tratamento medicamentoso com anti-hipertensivo há pelo menos seis meses, ter realizado última consulta com o médico da ESF há pelo menos seis meses, possuir pelo menos duas consultas médicas e duas de enfermagem no último ano e apresentar condições clínicas (físicas e psicológicas) para responder aos instrumentos.

Foram selecionados 172 usuários hipertensos, no entanto, quatro estavam internados, nove não foram localizados e quatro recusaram-se a fazer parte da pesquisa, obtendo-se um total de 155 participantes.

A coleta de dados foi iniciada após aprovação do projeto pelo Comitê de Ética em Pesquisa da Universidade Federal de Sergipe (CAAE - 0328.0.107.107-11).

As pessoas que concordaram participar da pesquisa foram orientadas a assinar o Termo de Consentimento Livre e Esclarecido (TCLE). A coleta foi realizada inicialmente consultando os prontuários. Em seguida, os pesquisadores entrevistaram de forma individual cada participante nas suas residências, iniciando pela caracterização sóciodemográfica e clínica dos participantes. Para avaliar a OV, foi utilizado o Medical Outcomes Study 36 - item Short-Form (SF-36), em sua versão traduzida e validada para o português do Brasil (9), com 36 questões agrupadas em oito componentes. A medida do questionário varia em uma escala de zero a 100; quanto maior a pontuação, melhor será a $Q V^{(9)}$.

Foi utilizado o Inventário de Depressão de Beck (BDI), em sua versão traduzida e validada para o português ${ }^{(10)}$, composto por 21 itens, cuja intensidade varia de zero (0) a três (3). O total possivel para a escala varia de zero a 63 , com maiores valores indicando maior depressão(11)

Utilizou-se o instrumento denominado Medida de Adesão aos Tratamentos (MTA) ${ }^{(12)}$, composto por sete itens que avaliam o comportamento do indivíduo em relação ao uso diário dos medicamentos. Os valores obtidos com as respostas aos sete itens são somados e divididos pelo número de itens, variando de um a seis, e posteriormente transformados numa escala dicotômica (Aderente/Não aderente) $)^{(13)}$.

Foram realizadas análises descritivas de frequência simples para variáveis nominais ou categóricas, de tendência central (média e mediana) e dispersão (desvio-padrão) para as variáveis contínuas. Também foram realizados testes estatísticos: teste de Mann Whitney para comparação das médias dos domínios do SF-36, do BDI e do MTA frente às variáveis: sexo, presença de companheiro (a), prática de atividade física e uso de medicação controlada; teste de correlação de Spearman para avaliar a correlação entre os domínios do SF-36, BDI e MTA com as variáveis: idade, renda

18 | Enferm. Foco 2016; 7 (2): 17-21 
familiar e número de medicações em uso.

Foi utilizada regressão linear simples para investigar a relação entre domínios do SF-36 e BDI com variáveis sociodemográficas. Foi utilizado o teste $\mathrm{T}$ de student para avaliar a significância das variáveis, bem como foram apresentadas as variáveis significativas em modelos multivariados quando houve. A consistência interna dos domínios do SF-36, do BDI e do MTA foi calculada utilizando a Alfa de Cronbach. O nivel de significância para os testes realizados foi de 0,05 e o software utilizado foi o $\mathrm{R}$ Core Team 2015.

\section{RESULTADOS}

Os participantes apresentaram idade média de 62,6 anos, a maioria era do sexo feminino (119, 76,8\%), possuía companheiro (a) (83, 53,5\%), declarava-se não negra $(71,7 \%)$ e tinha o 1 o grau completo ou incompleto (101, $65,2 \%)$. Com relação ao exercício de atividade laboral, dez $(6,5 \%)$ trabalhavam, 15 (9,7\%) estavam desempregados, 98 $(63,2 \%)$ aposentados ou eram pensionistas e 32 (20,6\%) se enquadravam em outras situações (trabalho em casa, afastado pelo INSS). A renda individual era de $\mathrm{R} \$ 548,9$ $\pm \mathrm{R} \$ 512,1$. A maior parte não praticava atividade física regularmente $(102,65,8 \%)$ e não fazia uso de medicação controlada (123, 79,4\%).

Foi calculada consistência interna dos instrumentos de medida utilizados. Os valores para os domínios do SF-36 variaram de 0,619 (Estado Geral de Saúde) a 0,942 (Aspectos Emocionais). O BDI apresentou valor igual a 0,81 e o MTA 0,78 .

A análise descritiva das medidas utilizadas para avaliar a OV, sintomas depressivos e adesão ao tratamento está apresentada na tabela 1 .

Tabela 1 - Análise descritiva dos domínios do SF-36, BDI e MTA. Aracaju - SE, 2012

\begin{tabular}{llll} 
Variáveis & Média (D.P) & Mediana & Intervalo \\
Componentes do SF-36 & & & \\
Aspecto Físico & $49,03(44,5)$ & 50 & $0-100$ \\
Dor & $59,8(28,2)$ & 61 & $0-100$ \\
Estado Geral de Saúde & $60,2(22,5)$ & 62 & $0-100$ \\
Capacidade Funcional & $62,3(29,4)$ & 65 & $0-100$ \\
Vitalidade & $62,4(23,3)$ & 65 & $0-100$ \\
Saúde Mental & $69,1(24,3)$ & 76 & $0-100$ \\
Aspecto Emocional & $75,0(41,0)$ & 100 & $0-100$ \\
Aspecto Social & $79,5(27,7)$ & 100 & $0-100$ \\
BDI & $12,3(8,1)$ & 11 & $0-48$ \\
MTA & $35,0(6,6)$ & 37 & $9-42$ \\
\hline
\end{tabular}

D.P.: Desvio Padrão
Quando foi avaliada a presença de sintomas depressivos a partir de estratos ${ }^{(11)}, 67$ (43,2\%) não os apresentaram, 54 (34,8\%) apresentaram disforia, oito (5,2\%) estado depressivo leve, 22 (14,2\%) depressão moderada, quatro (2,6\%) depressão grave.

Com relação à adesão ao tratamento medicamentoso, considerando a possibilidade de avaliação dos resultados utilizando escala dicotômica, 36 (23,2\%) não aderiam ao tratamento e 119 (76,8\%) aderiam.

Os homens apresentaram melhor avaliação em seis dos oito domínios do SF-36, Capacidade funcional, Dor, Estado geral de saúde, Aspecto emocional, Saúde mental e Vitalidade $(p<0,05)$. As mulheres apresentaram melhor avaliação nos domínios Aspectos físicos e sociais $(p<0,05)$. Com relação à avaliação do BDI e MTA frente ao sexo, as diferenças de média foram discretas, assim como na avaliação dos domínios do SF-36, do BDI e do MTA com o estado civil.

Quando avaliada a OV frente ao uso de medicação controlada, o grupo que não fazia uso apresentou melhor avaliação em todos os domínios, sendo essa diferença estatisticamente significativa nos domínios Aspectos emocionais e Saúde mental $(p<0,05)$. O grupo que fazia uso de medicação controlada apresentou maior valor médio no BDI, quando comparado ao que não fazia uso, e essa diferença foi estatisticamente significativa $(p<0,05)$. Já com relação ao MTA, as diferenças foram discretas.

Quanto à prática de atividade física, os que praticavam também apresentaram melhor avaliação em todos os domínios, sendo essa diferença estatisticamente significativa $(p<0,05)$ para seis dos oito domínios do SF36: Capacidade funcional, Aspectos físicos, Dor, Estado geral de saúde, Vitalidade e Saúde mental. O grupo que praticava exercício físico apresentou menor média no BDI, sugerindo menor probabilidade de apresentar sintomas depressivos $(p<0,05)$ e maior adesão, todavia a diferença no MTA foi mínima.

Foi utilizada regressão linear simples para investigar a relação entre domínios do SF-36 e BDI com variáveis sóciodemográficas (idade, sexo, escolaridade, estado civil, renda individual e trabalho). Foi utilizado o teste $T$ de student para avaliar a significância dos parâmetros, bem como foram apresentadas as variáveis significativas em modelos multivariados quando houve.

A tabela 2 demonstra os resultados significativos das regressões lineares para os domínios do SF-36 Capacidade funcional, Vitalidade, Aspecto emocional e Saúde mental, assim como para o BDI. 
Tabela 2 - Regressão Linear para Capacidade Funcional, Vitalidade, Aspecto Emocional, Saúde Mental e BDI Bruto. Aracaju, 2012.

\begin{tabular}{|c|c|c|c|c|c|}
\hline & \multicolumn{5}{|c|}{ IC $95 \%$} \\
\hline & B & EP & Inf & Sup & $\mathrm{p}$ \\
\hline \multicolumn{6}{|l|}{ Capacidade Funcional } \\
\hline Intercepto & 80,5 & 8,93 & 62,98 & 98,01 & $<0,001$ \\
\hline \multicolumn{6}{|l|}{ Trabalho } \\
\hline $\begin{array}{l}\text { Empregado } \\
\text { (Referência) }\end{array}$ & - & - & - & - & - \\
\hline Desempregado & 6,83 & 11,53 & $-29,44$ & 15,78 & 0,554 \\
\hline $\begin{array}{l}\text { Aposentado ou } \\
\text { Pensionista }\end{array}$ & $-23,71$ & 9,38 & $-42,10$ & $-5,33$ & 0,011 \\
\hline Outro & $-12,06$ & 10,23 & $-32,13$ & 8,00 & 0,239 \\
\hline \multicolumn{6}{|l|}{ Vitalidade } \\
\hline Intercepto & 59,87 & 2,09 & 55,77 & 63,98 & $<0,001$ \\
\hline \multicolumn{6}{|l|}{ Sexo } \\
\hline Masculino & 10,96 & 4,34 & 2,44 & 19,47 & 0,012 \\
\hline Feminino(Referência) & - & - & - & - & - \\
\hline \multicolumn{6}{|l|}{ Aspecto Emocional } \\
\hline Intercepto & 66,88 & 4,74 & 57,6 & 76,17 & $<0,001$ \\
\hline $\begin{array}{l}\text { Renda Individual } \\
(\mathrm{R} \$ 1,00)\end{array}$ & 0,015 & 0,006 & 0,002 & 0,027 & 0,019 \\
\hline \multicolumn{6}{|l|}{ Saúde mental } \\
\hline Intercepto & 62,29 & 2,76 & 56,88 & 67,69 & $<0,001$ \\
\hline $\begin{array}{l}\text { Renda Individual } \\
(\mathrm{R} \$ 1,00)\end{array}$ & 0,012 & 0,004 & 0,005 & 0,02 & 0,001 \\
\hline \multicolumn{6}{|l|}{ BDI Bruto } \\
\hline Intercepto & 16,08 & 1,53 & 13,07 & 19,09 & $<0,001$ \\
\hline \multicolumn{6}{|l|}{ Escolaridade } \\
\hline $\begin{array}{l}\text { Analfabeto } \\
\text { (Referência) }\end{array}$ & - & - & - & - & - \\
\hline Ensino fundamental & $-3,79$ & 1,72 & $-7,16$ & $-0,41$ & 0,03 \\
\hline Ensino médio & $-7,03$ & 2,33 & $-11,59$ & $-2,46$ & 0,003 \\
\hline Ensino superior & 7,92 & 7,98 & $-7,72$ & 23,56 & 0,321 \\
\hline
\end{tabular}

B - Parâmetro estimado; EP - Erro Padrão; IC - Intervalo de Confiança de 95\%; Inf - Inferior; Sup - Superior

A correlação entre as medidas SF-36, BDI e MTA também foi avaliada. Todos os domínios do SF-36 apresentaram correlação negativa com o BDI, que variou de moderada (Capacidade funcional $(r=-0,408, p=0,00)$ Aspectos físicos $(r=-0,374, p=0,00)$, Dor $(r=-0,349, p=0,00)$, Estado geral de saúde $(r=-0,466, p=0,00)$, Vitalidade $(r=-0,0444, p=0,00)$, Aspectos sociais $(r=-0,405, p=0,00)$, Aspecto emocional $(r=-0,442, p=0,00))$ a forte magnitude (Saúde mental $(r=-0,016, p=0,00))$. Quanto à avaliação do SF-36 com o MTA, as correlações foram positivas e fracas $(r<0,30)$, e do BDI com o MTA, negativas e fracas.

\section{DISCUSSÃO}

Algumas características sóciodemográficas da população se assemelham a de outros estudos como, prevalência do sexo feminino, idade, existência de companheiro(a) ${ }^{(4,6)}$ e baixa escolaridade ${ }^{(6)}$

Os indivíduos que fizeram parte da amostra apresentavam problemas para realizar atividades profissionais e/ou domésticas, devido sua saúde física (Capacidade funcional), assim como em outro estudo(5)

A ausência ou diminuição de atividade laboral está diretamente associada à capacidade funcional, com o grupo de indivíduos que estavam aposentados ou eram pensionistas, tendo apresentado diminuição da Capacidade funcional de 23,71 pontos, quando comparados aos que trabalhavam.

A média de idade dos indivíduos foi acima de 60 anos. Nesse sentido, um estudo que avaliou a capacidade funcional de idosos, tendo estabelecido como incapacidade funcional quando o idoso referia não conseguir realizar determinada atividade da vida diária, necessitando de ajuda de terceiros, identificou que a maioria dos idosos da amostra apresentou HAS e incapacidade funcional ${ }^{(14)}$.

A orientação sobre a importância da adoção de um estilo de vida saudável é fundamental no tratamento da HAS. Dentre essas recomendações, está a prática de atividade física ${ }^{(1)}$. No presente estudo, o grupo que praticava atividade física apresentou melhor avaliação da OV e menos sintomas depressivos. Nesse sentido, um estudo de revisão sistemática, também concluiu que existe associação positiva entre essas variáveis ${ }^{(15)}$.

Os homens apresentaram melhor OV considerando todos os domínios do SF-36. Esse resultado é semelhante ao apresentado em outro estudo realizado com 246 individuos hipertensos com algumas caracteristicas sóciodemográficas parecidas(b). ${ }^{(6)}$ domínio Vitalidade, que avalia a frequência com que cada indivíduo sente-se cheio de energia e vontade ou, por outro lado, cansado e esgotado, foi o que apresentou diferença estatisticamente significativa, influenciado pelo sexo, o que foi confirmado no modelo de regressão linear.

As mulheres vivem mais e apresentam mais comprometimento da saúde quando comparadas aos homens, assim como possuem mais dificuldade para desenvolver as atividades da vida diária, demonstrando maior incapacidade funcional, mais sintomas e QV inferior a dos homens ${ }^{(16)}$.

A renda individual interferiu na OV. Maior renda, melhor avaliação da qualidade de vida no Aspecto emocional e Saúde mental. Em outro estudo, entretanto, o domínio Capacidade funcional foi o que obteve melhor avaliação entre as pessoas com HAS que tinham melhor renda ${ }^{(6)}$. 
Aplicando estatística bivariada, foi observado que todos os domínios do SF-36 se correlacionaram com o BDI. O sinal negativo das correlações sugere que a presença de sintomas depressivos pode impactar negativamente a OV dos indivíduos com HAS que fizeram parte da amostra.

Quando avaliada a presença de sintomas depressivos e sua relação com o nível de escolaridade, indivíduos analfabetos apresentaram maior probabilidade de desenvolver sintomas de depressão quando comparados com os que cursaram ensino fundamental e médio. Tal resultado vem ao encontro de estudo cujos autores identificaram maior prevalência de depressão entre os indivíduos com menor escolaridade ${ }^{(17)}$.

Embora já tenha sido identificada correlação entre presença de sintomas depressivos e adesão ao tratamento medicamentoso(7), no presente estudo, essa correlação foi fraca $(r<0,30)$. Apesar do sentido ter sido negativo, sugere que a presença de sintomas depressivos diminui a adesão. As diferenças de resultados podem ser atribuídas às diferenças regionais e socioeconômicas dos indivíduos e metodológicas dos estudos.

\section{CONCLUSÃO}

O domínio da OV mais comprometido entre os participantes do estudo foi Aspecto físico. Para o sexo masculino, maior renda e desenvolver atividade laboral são variáveis relacionadas à uma melhor OV. A maioria apresentou alteração psicológica, que variou de disforia à depressão grave, assim como aderiu ao tratamento medicamentoso.

Os resultados desse estudo oferecem um diagnóstico do estado de saúde percebido pelos indivíduos e da sua adesão ao tratamento medicamentoso. Subsidiam, assim, o planejamento da assistência de enfermagem voltada a esse público, com vistas à promoção da saúde e prevenção de complicações e, por conseguinte, a uma melhoria da OV.

\section{REFERÊNCIAS}

1. Sociedade Brasileira de Cardiologia / Sociedade Brasileira de Hipertensão / Sociedade Brasileira de Nefrologia. VI Diretrizes Brasileiras de Hipertensão. Arq Bras Cardiol 2010; 95(1 supl.1): 1-51.

2. Brasil. Ministério da Saúde. DATASUS: indicadores de saúde. Disponivel em: http://www2.datasus.gov.br/DATASUS/index.php. Acessado em 17 de novembro de 2015 .

DATASUS [Internet]. Brasilia (DF): Ministério da Saúde. 2014 [citado 2015 mar 10]. Disponivel em: http://datasus.gov.br

3. Azevedo ALS de; Silva RA da; Tomasi, E; Quevedo L de Á. Doenças crônicas e qualidade de vida na atenção primária à saúde. Cad. Saúde Pública online]. 2013; 29(9):1774-82.

4. Ogunlana MO, Adedokun B, Dairo MD, Odunaiya NA. Profile and predictor of health-related quality of life among hypertensive patients in southwestern Nigeria. BMC Cardiovasc Disord. 2009: 17 (9):1-8.

5. Carvalho MAN, Silva IBS, Ramos SBP, Coelho LF, Gonçalves ID, Figueiredo Neto JA. Qualidade de Vida de Pacientes Hipertensos e Comparação entre dois Instrumentos de Medida de QVRS. Arq Bras Cardiol. 2012; 98(5):442-

6. Carvalho MV, Siqueira LB, Sousa ALL, Jardim PCBV. A influência da hipertensão arterial na qualidade de vida. Arq. Bras. Cardiol. 2013; 100 (2):164-74.

7. Krousel-Wood M, Islam T, Muntner P. Holt E, Joyce C, Morisky DE, Webber LS, Frohlich ED. Association of depression with antihypertensive medication adherence in older adults: cross-sectional and longitudinal findings from Cosmo. Ann Behav Med. 2010: 40(3):248-57.

8. Eze-Nliam CM, Thombs BD, Lima BB, Smith CG, Ziegelstein RC. The association of depression with adherence to antihypertensive medications: a systematic review. J Hypertens. 2010 Sep; 28(9):1785-95.

9. Ciconelli RM. Tradução para o português e validação do questionário genérico de avaliação de qualidade de vida "Medical Outcomes 36 Item Short Form Health Survey- SF-36" [Tese de Doutorado]. São Paulo: Escola Paulista de Medicina, Universidade Federal de São Paulo: 1997.
10. Gorenstein, C, Andrade, L. Inventário de depressão de Beck: propriedades psicométricas da versão em português. Rev Psiquiatr Clín. 1998; 25(5):245-50

11. Kendall PC, Hollon SD, Beck AT, Hammen CL, Ingrain RE. Issues and Recommendations Regarding Use of the Beck Depression Inventory. Cognit Ther Res. 1987; 11(3): 289-99.

12. Delgado AB, Lima ML. Contributo para validação concorrente de uma medida de adesão aos tratamentos. Psic, Saude \& Doenças. 2001; 2(2):81100 .

13. Gimenes HT, Zanetti ML, Haas VJ. Fatores relacionados à adesão do paciente diabético à terapêutica medicamentosa. Rev. Latino-Am. Enferm. 2009: 17(1):46-51.

14. Ferreira PC dos S, Tavares DM dos S, Rodrigues RAP. Características sociodemográficas, capacidade funcional e morbidades entre idosos com e sem declinio cognitivo. Acta Paul Enferm. 2011; 24(1):29-35.

15. Brasil. Ministério da Saúde. Secretaria de Vigilância em Saúde. Departamento de Vigilância de Doenças e Agravos não Transmissiveis e Promoção da Saúde. Vigitel Brasil 2014: vigilância de fatores de risco e proteção para doenças crônicas por inquérito telefônico / Ministério da Saúde, Secretaria de Vigilância em Saúde, Departamento de Vigilância de Doenças e Agravos não Transmissiveis e Promoção da Saúde. - Brasilia: Ministério da Saúde, 2015.

6. IBGE - Instituto Brasileiro de Geografia e Estatística. Sintese de Indicadores Sociais - Uma análise das condições de vida da população brasileira 2013. Ministério do Planejamento, Orçamento e Gestão. IBGE. Diretoria de Pesquisas. Coordenação de População e Indicadores Sociais. Estudos e Pesquisas, Informação Demográfica e Socioeconômica, 32. Rio de Janeiro, 2013

17. Molina MRAL, Wiener CD Branco JC, Jansen K, Souza LDM, Tomasi E et al. Prevalência de depressão em usuários de unidades de atenção primária. Rev. psiquiatr. clin. 2012; 39(6):194-7. 